\title{
Autonomous Vehicles: from Whether and When to Where and How
}

\section{Luciano Floridi $^{1,2}$}

Published online: 11 November 2019

(C) Springer Nature B.V. 2019

\section{Introduction}

When Death of a Salesman by Arthur Miller premiered in 1949, the job of selling merchandise by peddling wares in a designated area was common. It was a very different age - intermodal containers were being standardised, and consumerism was becoming rampant. In the play, the salesman, Willy Loman, is unhappy about all his travelling, and rightly so. The disappearance of his kind of job reminds us that, until recently, we thought that digital technologies were just going to decrease the need to move around. Today, we shop online, and the new selling agents are recommender systems (Milano et al. 2019), which canvass the space of information, or infosphere. It is true that we move a lot of bytes and not just atoms around, yet this is not the whole story. It is really too simplistic to conclude that the only impact that the digital revolution has, and is still having, on mobility has been that of reducing it. One still hears some driverless car evangelists arguing this (sometimes hijacking a green rhetoric) but they are clearly mistaken, because more people who cannot drive today will be able to do so in the future, thanks to increased levels of automation. It is far more accurate to say that digital technologies are changing the very essence of mobility (they are - re-ontologising it), in four different ways (see Fig. 1).

\section{Travelling, Journeys and Trips}

Every act of travel, understood as the movement of people from one geographical location to another, has two main components, which may be mapped on two axes (Fig. 1).

Luciano Floridi

luciano.floridi@oii.ox.ac.uk

1 Oxford Internet Institute, University of Oxford, 1 St Giles, Oxford OX1 3JS, UK

2 The Alan Turing Institute, 96 Euston Road, London NW1 2DB, UK 


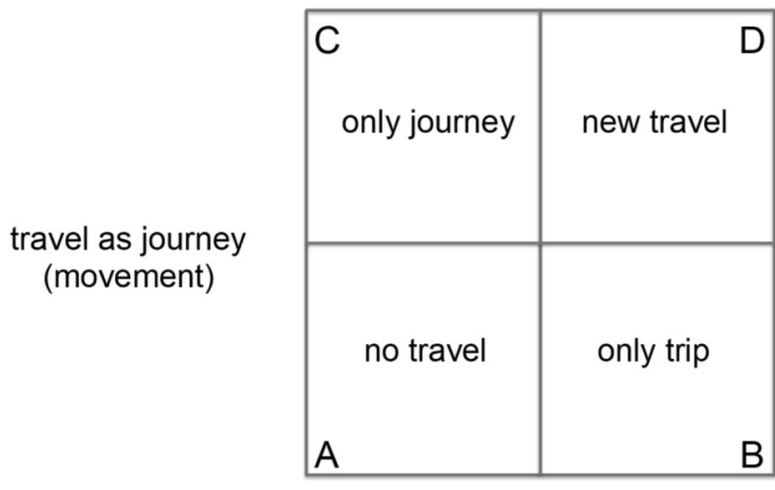

travel as trip

(experience)

Fig. 1 The two components of travel: the physical journey and the experiential trip

One is the physical process of movement, say from home to office and back. Let's call this $y$-axis, the physical journey. A journey has many aspects that are easily quantifiable, in terms of medium employed, costs, time, distance, speed, and so forth. The other component of travelling is the experience. Let's call this $x$ axis, the experiential trip. A trip has qualitative aspects that are more subjective and hence more difficult to specify, such as perceived duration, novelty, comfort, enjoyability, and so forth. The digital revolution has deeply transformed human mobility by changing both the journey and the trip component of travelling.

Consider $A$ first, in Fig. 1. This is the zero-travelling option I mentioned in Section 1. In some cases, digital technologies have eliminated both the journey and the trip because everything happens inside the infosphere. Telepresence ${ }^{1}$ and the remotization of jobs, for example, have hugely contributed to a lowering of the need for mobility. At the same time, option $A$ may simply shift the occurrence of travelling; our shopping online has caused a huge increase in delivery services, hence of journeys (the courier's), if not of trips (ours). This explains why lower human mobility leads to higher artificial mobility, that is, the replacement of people with artificial agents whenever possible, from delivery drones in the sky and luggage-sized vehicles navigating about town, to ships without crews. In other words, the more $A$ you have, the more $C$ you are likely to need. If this is correct, the future of drones lies not so much in transporting humans but in delivering goods, in tandem with AI solutions that can handle tasks such as navigation, orientation, scheduling, safety, and delivery.

At the opposite corner of the quadrant, in $D$, we find new forms of mobility made possible by the infosphere. Driverless motorbikes are a bit of an oxymoron not because motorbikes need us on board for balancing purposes, for this engineering problem can easily be solved (a self-balancing motorbike can park itself), but because motorbikes are often about the trip and the journey at least in equal measure, that is, they are often about the trip experience of the physical journey. Likewise, if you rent a sport car for a day, it is because you want to enjoy (trip) the driving (journey). This is why I doubt we

\footnotetext{
${ }^{1}$ For an epistemological analysis of telepresence, see Floridi (2005).
} 
shall see a driverless Ferrari. What the digital revolution has done is to make some mobility cheaper and safer (journey), and more enjoyable (trip). Thus, sustainable mobility trends made possible by ICTs, especially in urban contexts, such as 3rd generation bicycle-sharing systems, are a form of mobility that relies on a new combination of less impactful journeys that are, at least ethically speaking, more satisfactory trips.

Looking to the bottom right, we find in $B$ that digital technologies are trying to make any travel just a matter of enjoyable trip, eliminating as much as possible the tedious aspects of the journey. Cruises are a classic example; the journey is entirely absorbed by the trip. Consider on-board entertainments of all kinds in all sorts of vehicles, geolocation, digital maps, navigators, and other digital features: increasingly autonomous cars performing more and more functions independently of the driver, and these are all trends in the transformation of human mobility into a purely experiential phenomenon. In the future, one may imagine forms of virtual mobility becoming a reality in this context, e.g., replacing holiday tours as digital trips without physical journeys.

Finally, in the top left, in $C$, we find more efficient mobility, which tends to exclude more and more the human component: better and safer performance, lower costs, less time, better routes, just-in-time re-routing, re-scheduling, monitoring of consumption, better logistics, 24/7 services: these are some of the many aspects of a deep transformation of travel into unmanned journeys with no trip component, with AI systems in charge and humans at most 'on' the loop, placed in the $A$ square. Here, one of the great successes has been freight transport, which is increasingly automated, especially at sea. The foreseeable future includes the further automation of public transport in public spaces (e.g., dedicated lanes) and environment-bounded technology-friendly, local mobilities, such as airport buses, and robots in industrial logistics, as in warehouses. In this context, we should be careful not to confuse the logically possible with the actually feasible. In theory, level-5 autonomous vehicles - that is, those that are completely autonomous and require no driver-are perfectly (i.e. logically) possible because there is nothing intrinsically contradictory in assuming that, one day, all potential difficulties will be resolved by the right kind of technology. In practice though, what we are likely to witness will be deep transformation (re-ontologisation again) of whole environments to ensure that the available technologies will be successful (we are 'enveloping' the world around the capacities of our digital technologies, see Floridi (2014)). Think of the difference between (the logical possibility of) developing totally reliable visual systems to enable an autonomous vehicle to identify and recognise road signs in any weather condition in any context, from the snowy and foggy countryside to a rainy and traffic-bound city at night, to (the actual feasibility of) re-engineering all road signs in a given environment (say an airport) to make them communicate with the vehicle wirelessly and seamlessly, through radio signals, rather than visually.

\section{Not 'When' or Even 'Where' but 'How' is the Question}

Mobility is an essential component of life in any society. Every day, all over the world, billions of vehicles of all kinds (in particular, it is estimated that, since 
2010, more than 1 billion passenger cars travel the streets and roads of the world) play key functions (e.g., in transport or leisure) and social roles (e.g., as statements or status symbols). They are part of our history and culture. A transformation of mobility will affect the foundations of any society. And it is hard to imagine a more profound transformation of mobility than autonomous driving. This is why understanding attitudes towards the benefits and shortcomings of autonomous vehicles means being able to address societal welfare and individual well-being more successfully (Floridi et al. 2018). We saw in Section 2 that digital technologies have made it possible to detach the journey from the trip. It seems that, in the near future, we may be increasingly able to enjoy trips rather than journeys, with more freedom to choose to travel because we want to rather than because we need to. Willy Loman would have liked this. Here, I would like to introduce one more distinction that is equally important. A recent study (AUDI 2019) is very valuable because it provides a wealth of information and insights about people's attitudes to autonomous driving in China, France, Germany, Italy, Japan, South Korea, Spain, the UK and the USA. Studying its findings, it becomes clear that one should not confuse technological novelty with change.

The majority of those surveyed expressed interest (82\%) and curiosity (62\%) about autonomous driving. However, a majority also raised concerns about loss of control (70\%), technically unavoidable residual risks (66\%) and the lack of a legal framework (65\%). This is not as odd as it seems. Appreciating a novelty requires only an open mind but involves no actual risks or costs. Embracing a change implies a commitment that raises concerns about risks and costs (only $28 \%$ of people are willing to pay more for autonomous vehicles). Autonomous driving is both a realistic novelty and an unprecedented change. To translate high levels of interest and curiosity into low levels of concerns, one needs to provide better technology, more safety, and robust ethical and legal frameworks. Thus, high expectations about these latter variables are understandable.

Consider next that only a minority $(8 \%)$ 'feel able to explain the subject'. This may seem worryingly low and even cast doubts on the value of the survey. It is not, however, and it should not worry us. Take cars with automatic transmission. In 2018 , only $3.7 \%$ of the vehicles sold by CarMax (the largest used car retailer in the USA) had manual transmission. Cars with automatic transmission are by far the default option in the USA. Yet, arguably, only a very small percentage of drivers may 'feel able to explain' the difference between constantly variable transmission, dual clutch transmission, and simple automatic transmission. Attitudes are usually based on beliefs and experience rather than scientific knowledge. It would be a mistake to conclude that people's attitudes about something they cannot explain are insignificant or unreliable. What matters is that $90 \%$ of the people surveyed 'have heard of the technology' and $30 \%$ 'know it well'. A general conclusion that emerges from the survey may be summarised by a single word: variety. The question about the future of autonomous driving is not when or even where, but how it will take place. It will be a matter of what options, choices, and degrees of autonomous driving are offered to customers. Their needs, preferences, attitudes and circumstances differ. They are best addressed by a flexible variety of alternatives. 


\section{Conclusion}

In bad sci-fi movies, there are only new cars and a handful of models. Reality, however, is greasy and sticky, like a real engine. Public policies and business strategies about autonomous driving will need to make variety a feature, not a bug, and concentrate on re-engineering (enveloping) whole environments to make autonomous vehicles an ordinary reality. Hopefully, all this innovation will also ensure that autonomous vehicles will be environmentally more sustainable than the ones we drive today.

Acknowledgements I am very grateful to AUDI and the \&Audi initiative for the opportunity of collaborating on their study and for our many, informative meetings.

\section{References}

AUDI. 2019. The pulse of autonomous driving-an international user typology and an emotional landscape of autonomous driving. https://www.audi.com/en/company/research/and-audi-initiative/study-autonomousdriving.html.

Floridi, L. (2005). The philosophy of presence: from epistemic failure to successful observation. Presence Teleoperators and Virtual Environments, 14(6), 656-667.

Floridi, L. (2014). The fourth revolution-how the infosphere is reshaping human reality. Oxford: Oxford University Press.

Floridi, L., Cowls, J., Beltrametti, M., Chatila, R., Chazerand, P., Dignum, V., Luetge, C., Madelin, R., Pagallo, U., Rossi, F., Schafer, B., Valcke, P., \& Vayena, E. (2018). AI4People - an ethical framework for a good AI society: opportunities, risks, principles, and recommendations. Minds and Machines, 28(4), 689-707. https://doi.org/10.1007/s11023-018-9482-5.

Milano, Silvia, Mariarosaria Taddeo, and Luciano Floridi. 2019. Recommender systems and their ethical challenges. Available at SSRN 3378581.

Publisher's Note Springer Nature remains neutral with regard to jurisdictional claims in published maps and institutional affiliations. 